\title{
A Multi-Objective Optimization Model for Gas Pipeline Operations
}

\author{
Alem Demissie, Weihang Zhu \\ Department of Industrial Engineering, Lamar University, Beaumont, Texas 77710-0032
}

\begin{abstract}
Natural gas is usually transported by pipeline networks. This paper presents a multi-objective optimization model in optimizing the operation of natural gas pipeline networks. A mathematical model is established for different network configurations, namely linear, branch and looped topologies. Two conflicting objectives, power consumption minimization and gas delivery flow rate maximization, are considered subjected to pipeline and compressor constraints. The decision variables are the pressure at the nodes and the rotational speed of the compressors. A set of constraints is developed based on gas flow equations and compressors operating conditions. Due to the nonlinearity of the constraints and the objective, the developed model is a Nonlinear Programming problem. The optimization of the models is performed with NSGA-II algorithm. The solution obtained is a set of Pareto optimal points from which a decision maker can select a specific preferred solution. Sensitivity analysis was performed to determine the impact of parameter changes.
\end{abstract}

\section{Keywords:}

Natural gas, gas pipeline network, multi-objective optimization, NSGA-II 


\begin{tabular}{|c|c|c|c|}
\hline \multicolumn{4}{|c|}{ Nomenclature } \\
\hline$b$ & Supply/Delivery node & & Greek Abbreviations \\
\hline$C$ & Heat capacity $\left(\mathrm{Btu} / \mathrm{lbm}^{\circ} \mathrm{F}\right)$ & $\varepsilon$ & Pipe absolute roughness (inch) \\
\hline$f$ & Frictional factor & $\rho$ & Density $\left(\mathrm{lb} / \mathrm{ft}^{3}\right)$ \\
\hline$D$ & Pipe Diameter (inch) & $\omega$ & Rotational speed (rpm) \\
\hline$H$ & Compressor Head (lbf.ft/lbm) & $\eta$ & Efficiency \\
\hline$k$ & Isentropic exponent & & \\
\hline$L$ & Pipe Length (mile) & & Subscripts/Superscripts \\
\hline $\mathrm{n}$ & Number of moles & $c$ & Compressor \\
\hline$M$ & Molecular mass (g/mole) & $d$ & Discharge \\
\hline$\dot{m}$ & Mass flow rate $(\mathrm{lb} / \mathrm{min})$ & $g$ & Gas \\
\hline MAOP & $\begin{array}{l}\text { Maximum allowable operating pressure } \\
\text { (psi) }\end{array}$ & in & input \\
\hline$p$ & Pressure (psi) & $\max$ & Maximum \\
\hline$P$ & Power (HP) & $\min$ & Minimum \\
\hline$Q$ & Volume flow rate $\left(\mathrm{ft}^{3} / \mathrm{min}\right)$ & $p_{o}$ & Constant pressure (psi) \\
\hline $\bar{R}$ & $\begin{array}{l}\text { Universal gas constant } \\
\left(1545 \mathrm{ft} . \mathrm{lbf} / \mathrm{lbm} \mathrm{mol}^{\circ} \mathrm{R}\right)\end{array}$ & $s$ & Suction \\
\hline $\operatorname{Re}$ & Reynolds Number & $t$ & Turbine \\
\hline$S$ & Specific gravity & $i$ & Index, upstream node \\
\hline$T$ & Temperature $\left({ }^{\circ} \mathrm{R}\right)$ & $j$ & Index, downstream node \\
\hline$y$ & Mole fraction & is & isentropic \\
\hline$Z$ & Compressibility factor & out & delivery \\
\hline & & $U$ & Upper bound \\
\hline & & $L$ & Lower bound \\
\hline
\end{tabular}

\section{Introduction}

Natural gas plays a significant role as a clean and abundant energy resource in the global energy consumption. Natural gas is transported by pipeline networks which serve as the most cost effective transportation means over long distances. Natural gas transportation system consists of complex pipeline networks to transport natural gas from supply to demand regions. These pipelines are classified into gathering, transmission and distribution systems. Gathering systems transport raw natural gas from the wellhead to processing plants. Transmission systems transport high pressure gas over long distances from processing plants to distribution centers. Then, distribution systems transport natural gas at a reduced pressure to end consumers. Further classification of 
natural gas pipelines is based on network topologies. A linear topology corresponds to a linear arrangement of compressor stations. A tree topology corresponds to when compressors are arranged in branches and a cyclic topology is when compressors are arranged forming cycles with other compressor stations. Figure 1 shows examples of linear, tree and cyclic topologies.

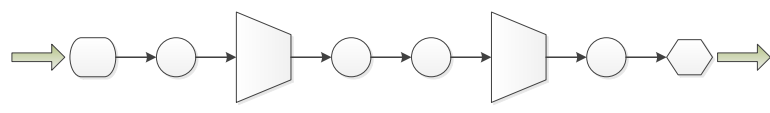

(a)

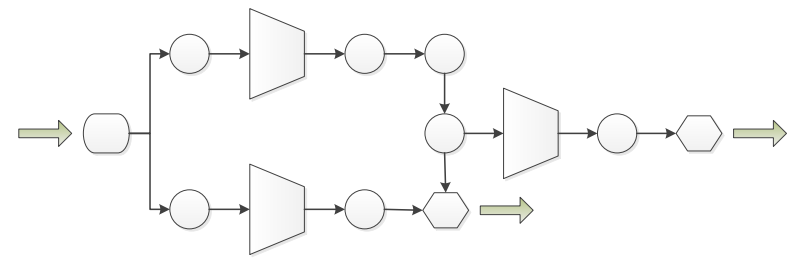

(c)

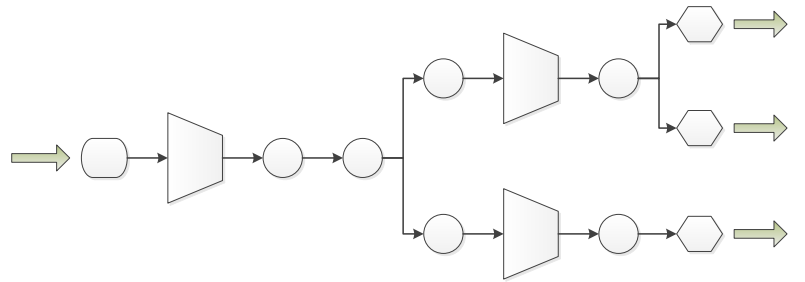

(b)

Legend

$\Longrightarrow$ Flow Direction

Supply Node
Compressor

Station

Demand Node $\longrightarrow$ Pipeline

Figure 1. Network topologies (a) Linear topology, (b) Tree topology and (c) Cyclic topology (Villalobos-Morales, Cobos-Zaleta, and Humberto J. Flores-Villarreal 2003)

As gas flows through pipelines, pressure drops due to friction between the gas and the walls of the pipeline. Compressor stations are installed along the pipeline to boost the gas pressure to ensure proper delivery. Compressor stations are pumping stations consisting of multiple compressor units, scrubber, cooling facilities, emergency shutdown systems, and an on-site computerized flow control and dispatch system that maintain the operation of the system (EIA 2007). These stations are usually coupled with gas turbines which consume part of the transported gas for operation. It is estimated that these stations consume 3-5\% of the gas and constitute $20 \%$ of the total operating cost of the company (S. Wu et al. 2000). It is also estimated that the operating cost of compressors represents between $25 \%$ and $50 \%$ of the total company's operating budget (F. Tabkhi 2007). Therefore, minimizing the fuel consumed by these stations has a tremendous economic benefit. 
Generally, optimization problems in natural gas pipeline networks are classified into design optimization and operation optimization. Design optimization involves the appropriate selection of layouts, materials and pipe sizes. It includes determining the types of compressors, power and distance between compressor stations to allow optimum operation and future expansion. The operation optimization of natural gas network deals with an existing network and station configuration. The goal is to find the optimal operating conditions of the network to minimize cost and satisfy customer demand. Design optimization and operation optimization can be further classified based on the objective functions as shown in Figure 2.

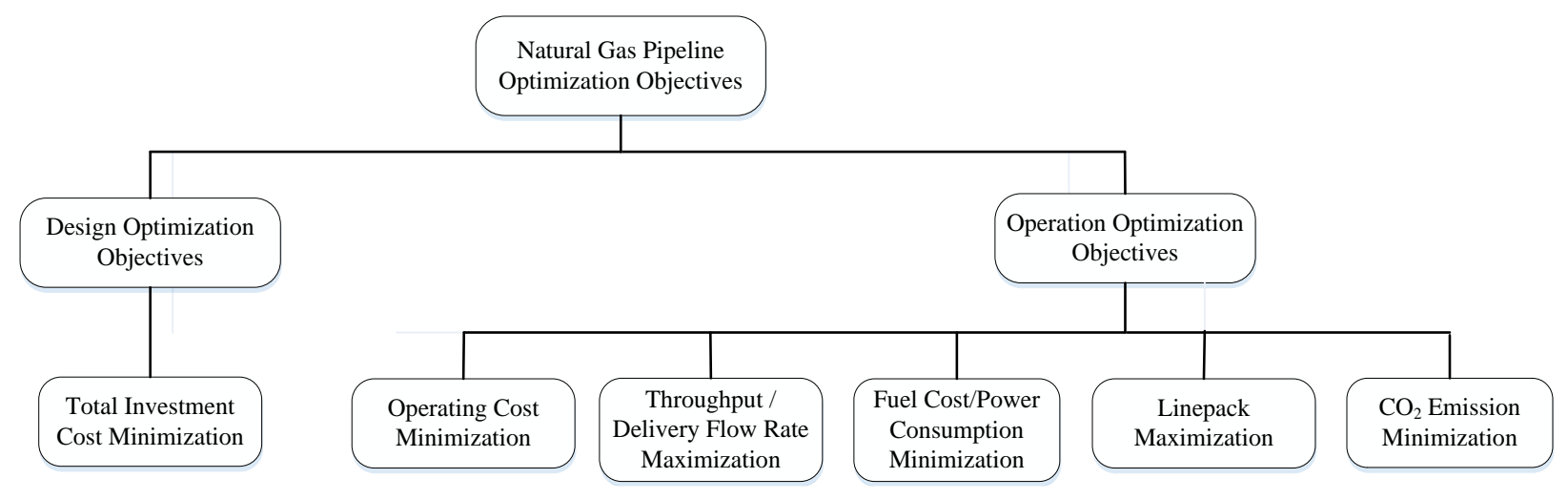

Figure 2. Classification of common optimization objectives in natural gas networks

\subsection{Literature Review}

Researchers have paid considerable attention to optimizing the design and operation of natural gas pipelines. Comprehensive literature survey papers have been conducted in the area of natural gas pipeline optimization to indicate the advancement in the field. Demissie and Zhu (2015) conducted a literature survey of natural gas pipeline design and operation optimization. Zheng et al. (2010) discussed the natural gas production, transportation and marketing models in the natural gas industry. Hamedi, Farahani, and Esmaeilian (2011) conducted a survey of optimization 
methods in natural gas design, flow, operation, and expansion systems. They addressed different network topologies and system assumptions. Ríos-Mercado and Borraz-Sánchez (2015) conducted a literature survey on line-pack, pooling, and fuel cost minimization problems. In this section, we will focus on fuel cost minimization and related Multi-objective Optimization of natural gas pipelines literatures which are most relevant to the goal of this research work.

\subsubsection{Fuel Cost Minimization}

The objective of the fuel cost minimization (FCM) problem is to minimize the fuel consumed by the compressor stations in natural gas transmission pipelines subjected to pipeline and compressor constraints. There are several variations of the FCM problem depending on the modeling assumptions and the decisions to be made. Basically, the research conducted on FCM can be classified into nonlinear programing (NLP) and mixed integer nonlinear programming (MINLP) problems.

The NLP model takes into account two types of continuous decision variables: mass flow rate through each arc and gas pressure level at each node (De Wolf and Smeers 2000). It assumes that the number of compressor units to be operated within in each station is known in advance. Besides the continuous decision variables, MINLP models consider discrete decision variables that represent the number of compressors to be turned on. Practically, the nature of FCM problem is very complex to solve computationally due to the non-linearity and non-convexity of the model.

\section{Nonlinear Programming (NLP)}

Wu et al. (2000) performed an in depth study of the mathematical model of the FCM problem considering identical centrifugal compressors arranged in parallel. They derived a lower bounding 
scheme based on model relaxations for the compressor domain and fuel cost function to reduce the problem dimension. Villalobos and Ríos-Mercado (2002) extended the works of Wu et al. (2000) by developing approximation functions using data from nine compressor units. Christo et al. (2008) adapted the FCM model developed by Wu et al. (2000) for compressor stations composed of compressors not necessarily identical. De Wolf and Smeers (2000) used piecewise linear approximations for the nonlinear flow-pressure relations and solved the problem by an extension of the simplex method. Carter et al. (2000) addressed fuel consumption or electric power minimization problem. They considered inlet and outlet flow as decision variables and compared sampling algorithm methods such as implicit filtering, DIRECT and a hybrid of the two.

Network problems with loops are more complex to solve because the flow variables cannot be uniquely determined. Kim, Ríos-Mercado, and Boyd (2000) applied heuristics to solve FCM problem for cyclic networks. They initially fixed gas flow variables and found pressure variables by dynamic programming (DP) and then fixed pressure variables and found flow variables. RíosMercado (2002) addressed both non-looped and looped network topologies. They applied DP for non-looped networks and NDP for looped systems. Flores-Villarreal and Ríos-Mercado (2003) also considered linear, tree and cyclic network topologies and solved the problem using Generalized Reduced Gradient method. Borraz-Sánchez and Ríos-Mercado (2005) proposed a hybrid heuristic solution procedure based on a non-sequential dynamic programming and tabu search for cyclic network topologies. Ríos-Mercado (2002) applied a combination of graph theory and nonlinear functional analysis. Borraz-Sanchez and Rios-Mercado (2004) solved the problem in two phases. First, they found a set of feasible flows by a reduction technique using preprocessing procedure. Then, they found an optimal set of pressures by applying NDP. Conrado Borraz- 
Sánchez and Haugland (2011) constructed a tree decomposition of the network and applied DP to solve the discrete part of the model.

\section{Mixed Integer Nonlinear Programming (MINLP)}

Cobos-Zaleta and Ríos-Mercado (2002) developed an MINLP model for the FCM problem. They used an outer approximation with equality relaxation and augment penalty method to handle the non-convexity of the problem. Villalobos-Morales, Cobos-Zaleta, and Humberto J. FloresVillarreal (2003) suggested the applications of preprocessing techniques such as variable bounding, initial variable value assignment and scaling for NLP and MINLP FCM models to find better local optima. Chebouba et al. (2009) considered the number of turbo compressors and discharge pressure as decision variables and used an ant colony optimization technique to solve the MINLP model developed. Firooz Tabkhi et al. (2010) developed an NLP model for simple linear network topology and MINLP model considering flow directions for multi-supply multidelivery transmission network. Sanaye and Mahmoudimehr (2012) addressed the limitations of NDP for cyclic networks and proposed a modified NDP and genetic algorithm method for different network topologies. Xu et al. (2014) adopted bundle projection subgradient combined with Lagrange multiplier method to solve the FCM problem. They found out that the optimization strategy alleviated the oscillation and speeded up convergence. Liu, Li, and Yang (2014) considered outbound pressure and number of compressors as decision variables to minimize the energy consumption of natural gas and solved the model using DP. 


\subsubsection{Multi-objective Optimization of Gas Pipeline Network}

The optimization of a natural gas transportation network is naturally a multi-objective optimization problem. However, it is mostly treated as single objective for the sake of simplicity. This is because multi-objective optimization algorithms are complex and computationally expensive. Hence, typically the most important objective is chosen as an objective function and the other objectives are often converted to be constraints by restricting their values within a certain range. Few literatures are found dealing with multi-objective optimization of natural gas pipelines.

Stoffregen et al. (2005) identified the optimum compressor, control valve and block valve operation of natural gas network system by the application genetic algorithm. Babonneau, Nesterov, and Vial (2012) formulated the operation and design of natural gas system as a biobjective problem. They considered minimizing the investment cost and minimizing energy cost as objective functions. Hernandez-Rodriguez (2011) addressed a tri-objective problem in natural gas operation system. X. Wu et al. (2014) focused on maximizing operation benefit and maximizing throughput. The developed model was solved using weighted sum method. Alinia Kashani and Molaei (2014) applied NSGA-II to minimize operating cost, maximize throughput and line pack.

It is noted that the majority of the literature in the area of optimization of natural gas pipelines addressed the problem as a single objective optimization problem. It typically considered the fuel cost minimization problem as a single objective for a given mass flow delivery.

The main contribution of this paper is to address the operation of natural gas pipeline as a multi-criteria decision making process and finding optimal operating conditions of two conflicting objectives, namely power consumption minimization and gas delivery flow rate maximization for 
three different network topologies. It also performs sensitivity analysis of the change in the objective function when decision variables vary.

The rest of the paper is organized as follows. Section 2 defines the mathematical model and considers case studies with different network topologies. Section 3 discusses problem solutions with multi-objective optimization and sensitivity analysis. Finally, the paper is concluded in Section 4.

\section{Modeling}

Part of natural gas in a pipeline network is consumed by compressor stations. Reducing the power consumed by the compressor stations helps in lowering fuel consumption and minimizes the energy cost thereby increasing the profit of a company. It also has a substantial environmental benefit by reducing the levels of greenhouse gas emissions to the atmosphere. In reality, the demand of natural gas varies according to environmental conditions and industrial requirements. This means the demand is seasonal. It remains steady for an interval. Thus, the operator need to adjust the optimum operating conditions for the required demand.

Therefore, the major challenge is to minimize the power consumption of the compressor stations and meanwhile maximize the amount of gas delivered to customers. This requires a balance between two conflicting objectives, namely minimizing the power consumed by the compressor stations and maximizing the gas delivery of the pipelines. As such, the decision making process of natural gas pipeline operation is a multi-criteria decision making process. Solving this problem as bi-objective optimization problems allows us to better and more quickly answer the questions from what-if analyses such as: 1) If we have an upper limit of allowable gas consumption by compressors, what will be the maximum amount of gas delivery through this 
network by adjusting operating parameters; and 2) If we need to meet a certain customer demand requirement, how to adjust operating parameters to minimize the gas consumed by compressors.

\subsection{System Assumptions}

Natural gas networks are complex transportation systems. Therefore, in the modeling of these networks, it is customary to make simplifications and break the networks into nodes, pipelines and compressor stations. The network comprises of supply nodes where gas is injected into the system, demand nodes where gas flows out of the system, and intermediate nodes where the gas is rerouted. Pipeline segments are represented by arcs between two nodes.

The following system assumptions are made:

Steady State: It is a well-established fact that flow in gas pipelines is unsteady (transient) in which flow parameters are always changing with time. However, transient analysis would require increasing the number of variables and high complexity of the problem. Therefore, it is convenient to make a simplifying assumption that the flow is steady. Under many conditions, this assumption produces acceptable results (Osiadacz and Chaczykowski 2001).

Isothermal flow: Basically, the temperature of the gas in buried pipeline varies along the length of the pipe due to heat transfer between the gas and the surrounding soil. However, if the pipeline is fairly long, the temperature will ultimately equal the ground temperature as gas approaches the delivery point. Therefore, the assumption of isothermal flow is good enough, since in long transmission lines the gas temperature reaches constant value equal to or close to the ambient temperature (Menon 2005). Hence, the operating temperature, $T$ is assumed to be constant and is $520^{\circ} \mathrm{R}$. 
Horizontal pipeline: It is assumed that there is no elevation difference between upstream and downstream points and as a result, the pipe segment is assumed to be horizontal.

Flow direction: Each pipeline segment (arc) in the network has a pre-specified direction.

Constant compressibility factor: The compressibility factor is the gas deviation factor from ideal gas. This value is calculated based on average upstream and downstream pressure and other flow parameters. In this research work, the pressure drop in the pipeline is approximately 10-30\%. Therefore, a constant average value of $Z=0.95$ is used in this study while in more advanced computation algorithms, $Z$ should be a variable value.

Identical compressors: Each compressor station consists of identical centrifugal compressors arranged in parallel. This type of compressor station is considered because it is very common in today's gas industry since it has relatively low maintenance and operation cost. The number of compressor units operating within each compressor station is assumed to be known in advance.

Isentropic compression: Reversible adiabatic compression is assumed.

\subsection{Governing Equations}

The mathematical model to represent a steady state flow of gas in a network is developed based on pipeline flow equations, principles of conservation of mass and performance characteristics and equations of compressors.

\subsubsection{Pipeline Equations}

Pipeline flow equation: The flow equation relates the gas flow rate with gas properties, pressure, pipe diameter and length. For a horizontal pipe, assuming temperature and compressibility factor stay constant, the steady-state pressure drop can be calculated as 


$$
Q_{j}^{2}=3.629 \frac{D_{i j}}{Z S_{g} T f_{i j} L_{i j}}\left(p_{i}^{2}-p_{j}^{2}\right)
$$

Specific Gravity: An important parameter is the specific gravity of the gas that is a measure of how heavy the gas is compared to air at a particular temperature and is calculated as

$$
S_{g}=\frac{M_{\text {gas }}}{M_{\text {air }}}
$$

The molecular mass of the gas is calculated by mixing rule as

$$
M_{g a s}=\sum M_{i} y_{i}
$$

Frictional factor: The frictional factor is an empirical parameter used to describe the energy loss due to friction between the walls of the pipe and the gas. Most natural gas pipelines operate in turbulent fully rough pipelines and hence, the frictional factor is estimated using Nikuradse's equation as

$$
\frac{1}{\sqrt{f_{i j}}}=-2 \log _{10}\left(\frac{\varepsilon_{i j}}{3.71 D_{i j}}\right)
$$

Maximum allowable operational pressure: The basic requirement of a gas pipeline is to transport the gas safely and economically. A key safety requirement is the determination of a pipeline's MAOP which is the highest pressure at which a pipeline can be operated such that

$$
p_{i j} \leq M A O P_{i j}
$$

\section{Continuity Equation}

The principle of continuity equation is derived from the fact that mass is always conserved in fluid systems regardless of the pipeline complexity or direction of flow. For a steady state system, the flow rate does not change over time. The mass flow rate is calculated as

$$
\dot{m}_{i j}=\rho_{j} Q_{j}
$$




\subsubsection{Compressor Equations}

The amount of energy supplied to the gas by the compressors depends on the pressure and flow rate of the gas. For a given pipeline, the head developed by the compressor is the amount of energy supplied to the gas per unit mass of the gas. It is an index of the ratio of discharge pressure and suction pressure and can be interpreted as the fictive height on which the gas has to be pumped to attain the same power. The value of the head can be calculated as:

$$
H=Z R T \frac{k}{k-1}\left[\left(\frac{p_{d}}{p_{s}}\right)^{\frac{k-1}{k}}-1\right]
$$

The isentropic exponent can be calculated as

$$
k=\frac{\sum C_{p i} M y_{i}}{\sum\left(C_{p i} M y_{i}\right)-\bar{R}}
$$

Therefore, the power required to compress the gas in the compressor is calculated as

$$
P=\frac{\dot{m}_{c} H}{\eta_{i s}}
$$

Note that as the flow rate increases, the pressure also increases and hence the power required will also increase.

Compressors provide the necessary pressure to the gas and at the same time operate within the operating limits. A natural way to characterize the performance of centrifugal compressors is in terms of the inlet volumetric flow rate, speed, adiabatic head, and adiabatic efficiency. The relationships between these quantities are usually represented by performance maps which are plots of $H$ and as functions of $Q$ at variety of speeds as shown in Figure 3 and Figure 4. 


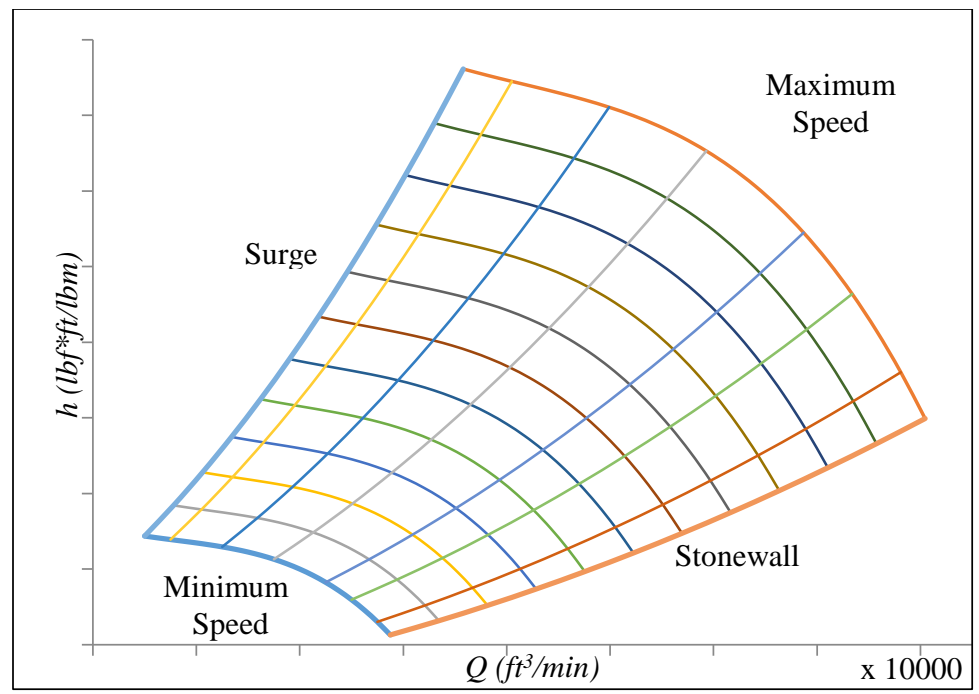

Figure 3. Centrifugal compressor map $H$ vs. $Q$ and $\omega$ (S. Wu et al. 2000)

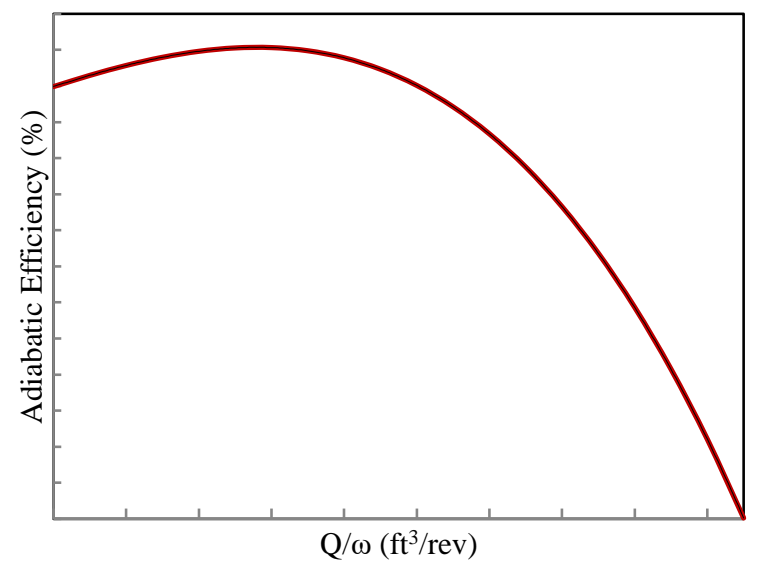

Figure 4. Adiabatic efficiency $\eta_{i}$ as a function of $Q / \omega(\mathrm{S}$. Wu et al. 2000)

These performance maps are approximated by cubic polynomial functions with constant coefficients and are fitted by least square method as shown in equations (10) and (11). 


$$
\begin{gathered}
\frac{H}{\omega^{2}}=A_{H}+B_{H}\left(\frac{Q_{s}}{\omega}\right)+C_{H}\left(\frac{Q_{s}}{\omega}\right)^{2}+D_{H}\left(\frac{Q_{s}}{\omega}\right)^{3} \\
\eta_{i s}=A_{E}+B_{E}\left(\frac{Q_{s}}{\omega}\right)+C_{E}\left(\frac{Q_{s}}{\omega}\right)^{2}+D_{E}\left(\frac{Q_{s}}{\omega}\right)^{3}
\end{gathered}
$$

where, $A_{H}, B_{H}, C_{H}, D_{H}$ and $A_{E}, B_{E}, C_{E}, D_{E}$ are constants which depend on the compressor unit. These equations vary for different compressors and are valid in the operational range of the compressor. The operational range of a compressor is limited by the maximum and minimum allowable speed and surge and stone wall limits as shown in Figure 3.

The minimum and maximum allowable rotational speed limits are indicated at the top and bottom side of Figure 3 and can be written as

$$
\omega_{\min } \leq \omega \leq \omega_{\max }
$$

The surge limit is depicted at the left side and the stonewall limit is at the extreme right side of Figure 3. These limits can be determined using equation (12).

$$
\left(\frac{Q_{L}}{\omega_{\min }}\right)_{\text {Surge }} \leq \frac{Q}{\omega} \leq\left(\frac{Q_{U}}{\omega_{\max }}\right)_{\text {Stonewall }}
$$

\subsection{Case Studies for Three Network Topologies}

Three case studies with some modifications have been adopted from the works $\mathrm{Wu}$ et al. that consider linear, branched and looped topologies (S. Wu et al. 2000). The goal is to find optimum operating condition for any given topology.

The composition of natural gas and its thermodynamic properties are given in Table 1. 
Table 1. Parameters of the components of the gas flowing in the pipeline (S. Wu et al. 2000)

\begin{tabular}{llll}
\hline Component & Methane & Ethane & Nitrogen \\
\hline Mole percent & 85 & 14 & 1 \\
Molecular weight & 16.04 & 30.07 & 28.01 \\
Heat capacity at constant pressure & 0.59 & 0.39 & 0.25 \\
\hline
\end{tabular}

The molecular mass and the specific gravity of the gas are calculated using equation (3) and (2) respectively.

The fitted coefficients of the polynomial functions for isentropic head and isentropic efficiency stated in equations (10) and (11) of the compressor performance characteristic are

$$
\begin{array}{ll}
A_{H}=0.6824 \times 10^{-3} & A_{E}=134.8055 \\
B_{H}=-0.9002 \times 10^{-3} & B_{E}=-148.5468 \\
C_{H}=0.5689 \times 10^{-3} & C_{E}=125.1013 \\
D_{H}=-0.1247 \times 10^{-3} & D_{E}=-32.0965
\end{array}
$$

The minimum speed and maximum speed of the compressors are $5000 \mathrm{rpm}$ and $9400 \mathrm{rpm}$. The upper and lower volumetric flow rates of the compressors are $7000 \mathrm{ft}^{3} / \mathrm{min}$ and $22000 \mathrm{ft}^{3} / \mathrm{min}$ respectively. Hence, the upper and lower bounds of the head of the compressor can be calculated using equation (10) and the isentropic efficiency of the compressors is calculated using equation (11). The surge and stonewall can be obtained from equation (12).

\section{Case 1. Linear Topology}

A single supply single delivery network consisting of a two compressor stations is considered. Each compressor station has six identical compressor units arranged in parallel as shown in Figure 
5. The network has three pipelines and the diameter of the pipelines is 24 inches. The upper and lower pressure limits in the nodes are 600 and 800 psia respectively.

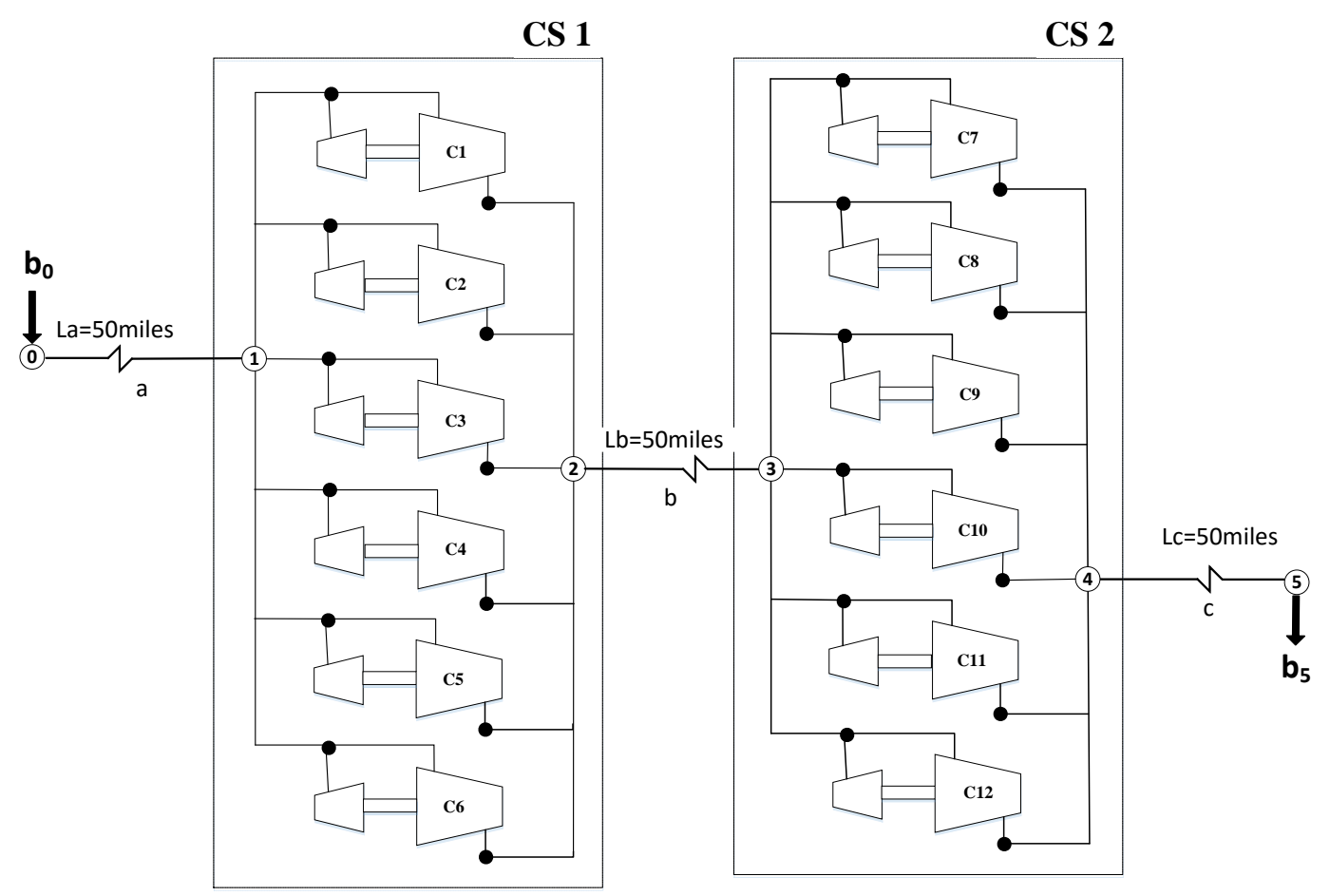

Figure 5. Case study 1: linear network topology

\section{Case 2. Branched Topology}

In the second case study, a branched network consisting of three compressor stations as shown in Figure 6 is considered. The first compressor station has 6 compressors and the second and third stations have 3 identical compressors. The upper and lower pressure limits at the nodes are 600 and 800 psia respectively. The network has seven pipelines with a diameter of 24 inches. 


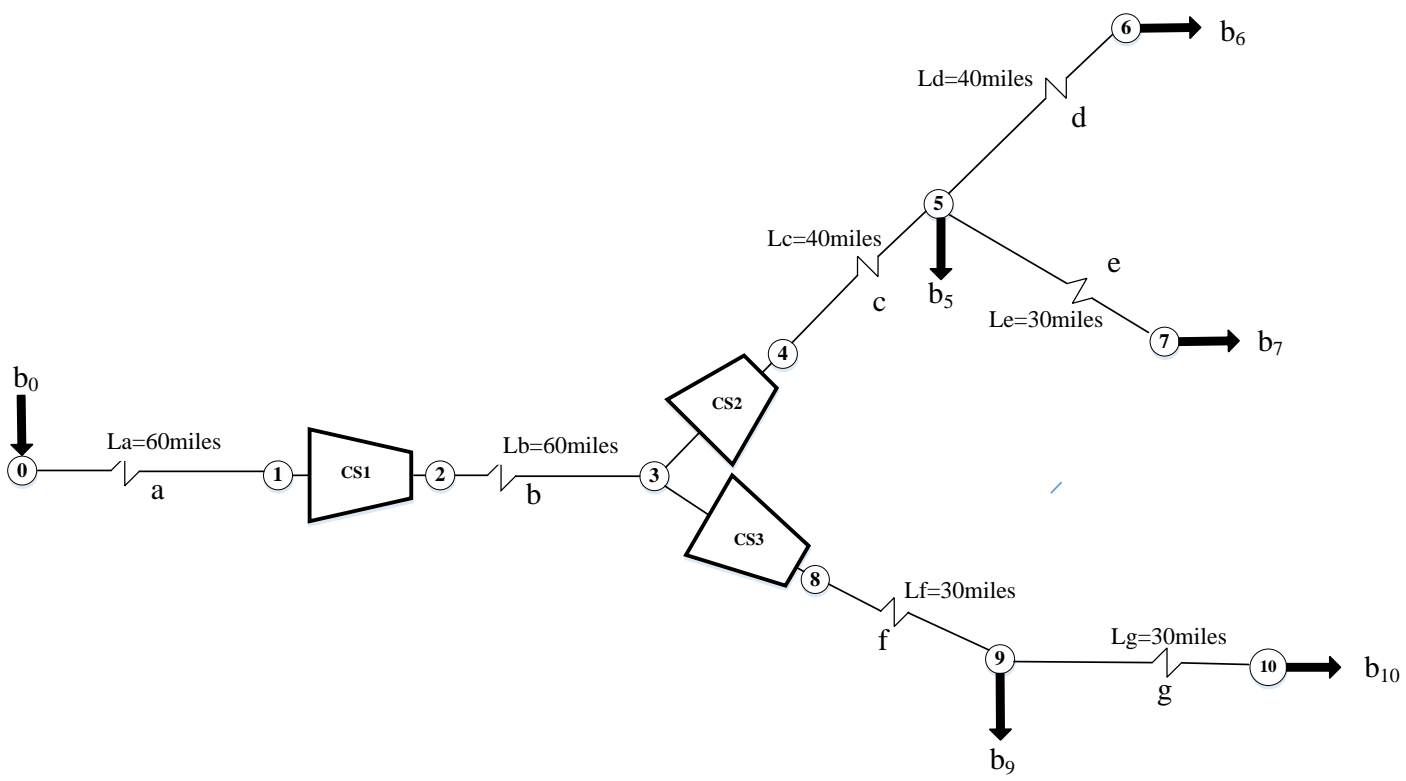

Figure 6. Case study 2: branched network topology

\section{Case 3. Looped Topology}

A pipe network with single loop consisting of two compressor stations is considered as shown in Figure 7. Each compressor station has five identical compressors arranged in parallel. The network has six long pipelines and the minimum and maximum allowable pressure in the pipelines are 600 psia and 800 psia respectively. 


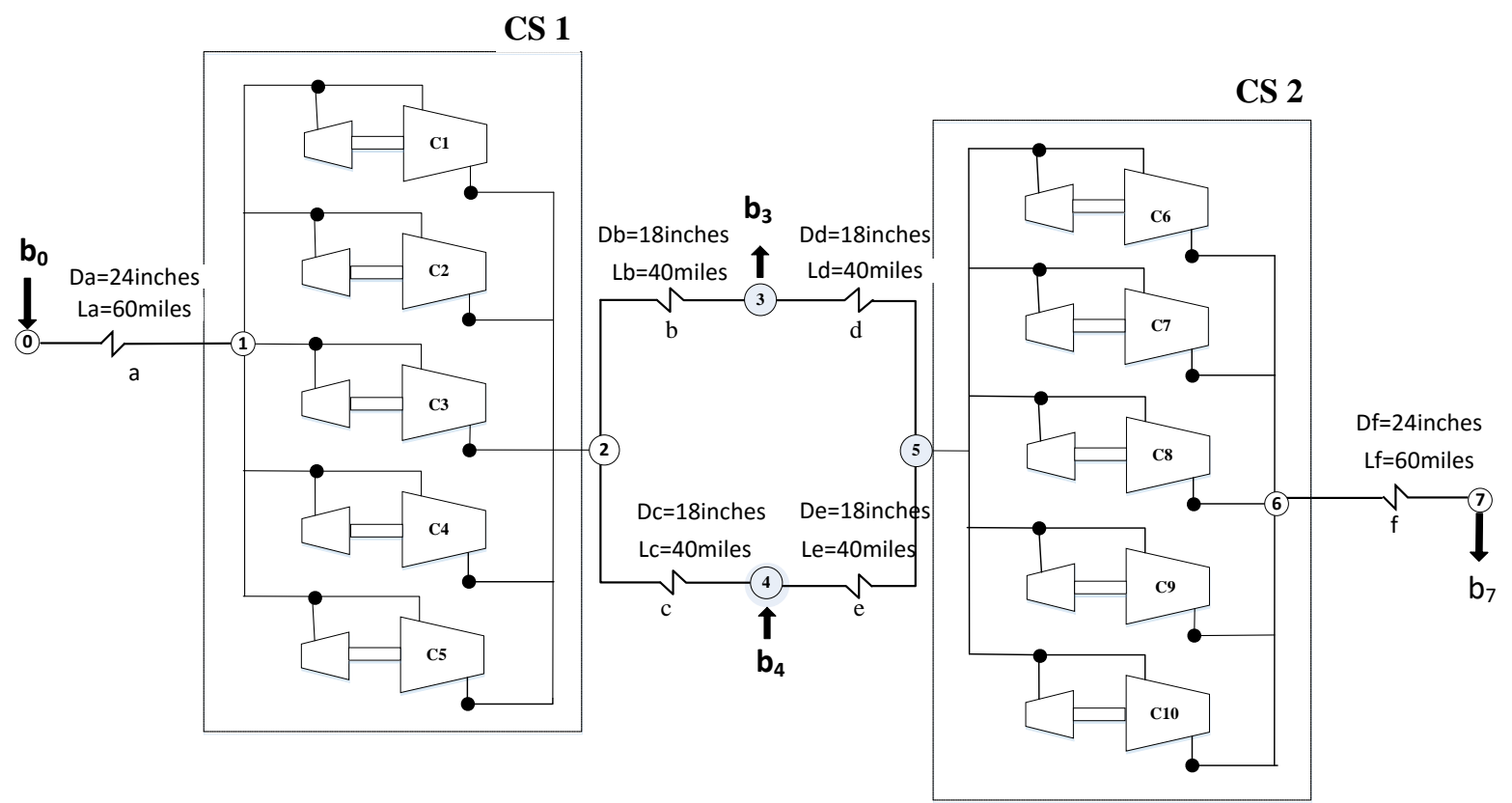

Figure 7. Case study 3: looped network topology

\subsubsection{The Multi-objective Optimization Model}

\section{Decision variables}

The basic continuous decision variables are the pressure at the nodes, $p_{i}$ and the rotational speed of compressors, $\omega_{i}$

\section{Two Objective Functions}

i. Maximize the gas delivery flow rate of the system

$$
\text { Maximize } \sum b_{\text {out }}
$$

ii. Minimize the power consumption of compressor stations

$$
\text { Minimize } K \sum \frac{b_{\text {out }} H}{\eta}
$$

$\mathrm{K}$ is a constant that depends on the fraction of flowrate through an individual compressor station. 


\section{Constraints}

- Flow balance equation at each node under steady state condition.

$$
\sum \dot{m}_{\text {in }}+\sum b_{\text {in }}=\sum \dot{m}_{\text {out }}+\sum b_{\text {out }}
$$

- Pipe flow equation in each pipe segment under steady state condition using equation (1).

- Lower and upper bound of pressure variables.

$$
p_{i}^{L} \leq p_{i} \leq p_{i}^{U}
$$

- Nonconvex feasible operating domain of each compressor unit provided in equations (10) and (11).

- Maximum and minimum rotational speed of the compressors.

$$
\omega_{\min } \leq \omega_{i} \leq \omega_{\max }
$$

- Surge and stonewall limits.

$$
\frac{Q_{L}}{\omega_{\min }} \leq \frac{Q_{i}}{\omega_{i}} \leq \frac{Q_{U}}{\omega_{\max }}
$$

\section{Results and Discussions}

In the previous section, a multi-optimization model was developed to minimize power consumption of compressor stations and maximize gas delivery flow rate of gas pipelines. The nature of the model is non-linear and possesses conflicting objectives and highly complex search space. Hence, the problem gives rise to a set of trade-off optimal solutions, popularly known as Pareto-optimal solutions. There are a number of meta-heuristics methods to approximately solve 
non-linear optimization problems. Among these methods stochastic evolutionary algorithms have gained popularity due to their suitable nature in the application of multi-objective optimization of NLP problems.

In this research work, the optimization of the model is performed using NSGA-II which is an evolutionary multi-objective optimization procedure that attempts to find multiple Pareto-optimal solutions. It uses the non-dominated sorting scheme and crowding measure to rank individual designs (Deb 2001). The algorithm is run using jMetal (Metaheuristic Algorithms in Java) which is an object-oriented Java-based framework for solving multi-objective optimization problems (Durillo and Nebro 2011). In the implementation of NSGA-II, a population size of 200 is considered. The maximum number of objective function evaluation allowed is set to be 25,000 and the probability of the crossover is 0.9 . The maximum number of iterations allowed is set to be 100.

Case 1 which is a linear topology considers 8 decision variables and 23 constraints to optimize the objective functions. The optimization problem is solved and the Pareto optimal points for gas delivery flow rate maximization and power consumption minimization are presented in Figure 8. The CPU computation time for this case is averaged as 5,848ms. 


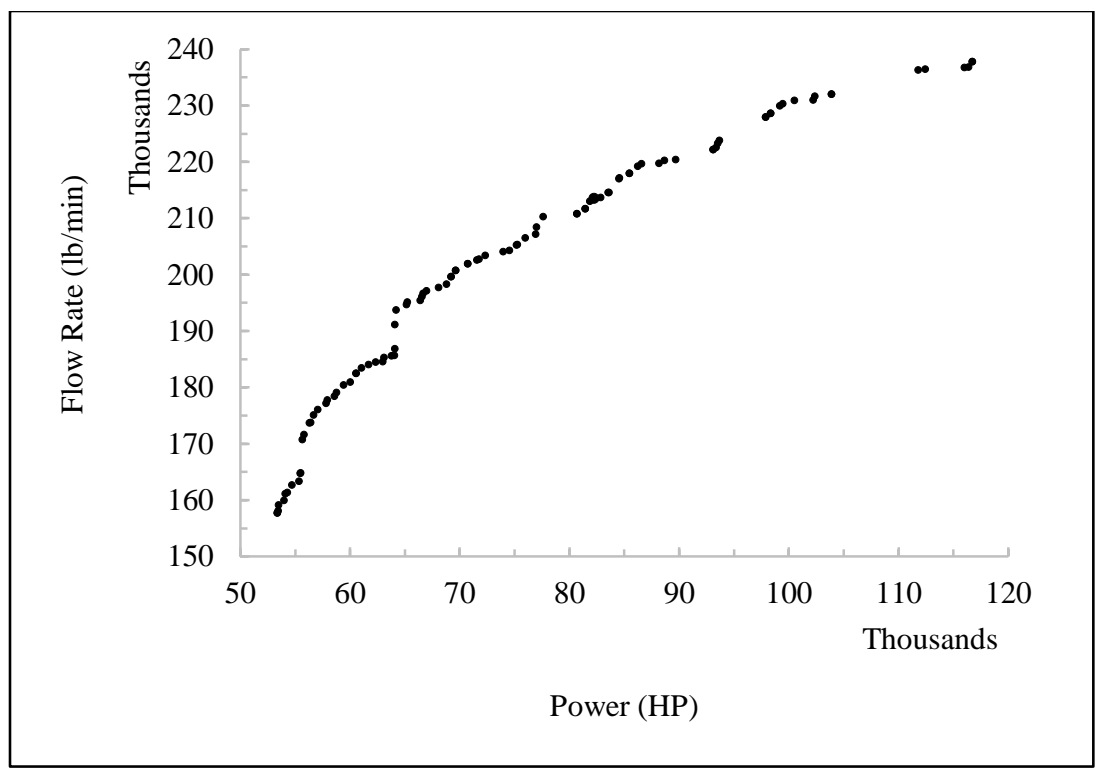

Figure 8. Optimum points for minimizing power consumption and maximizing delivery flow rate for linear topology

Case 2 which is a branched topology considers 14 decision variables and 40 constraints to optimize the objective functions. The optimization problem is solved and the Pareto optimal points for gas delivery flow rate maximization and power consumption minimization are presented in Figure 9. The CPU computation time for this case is averaged as 7,290ms. 


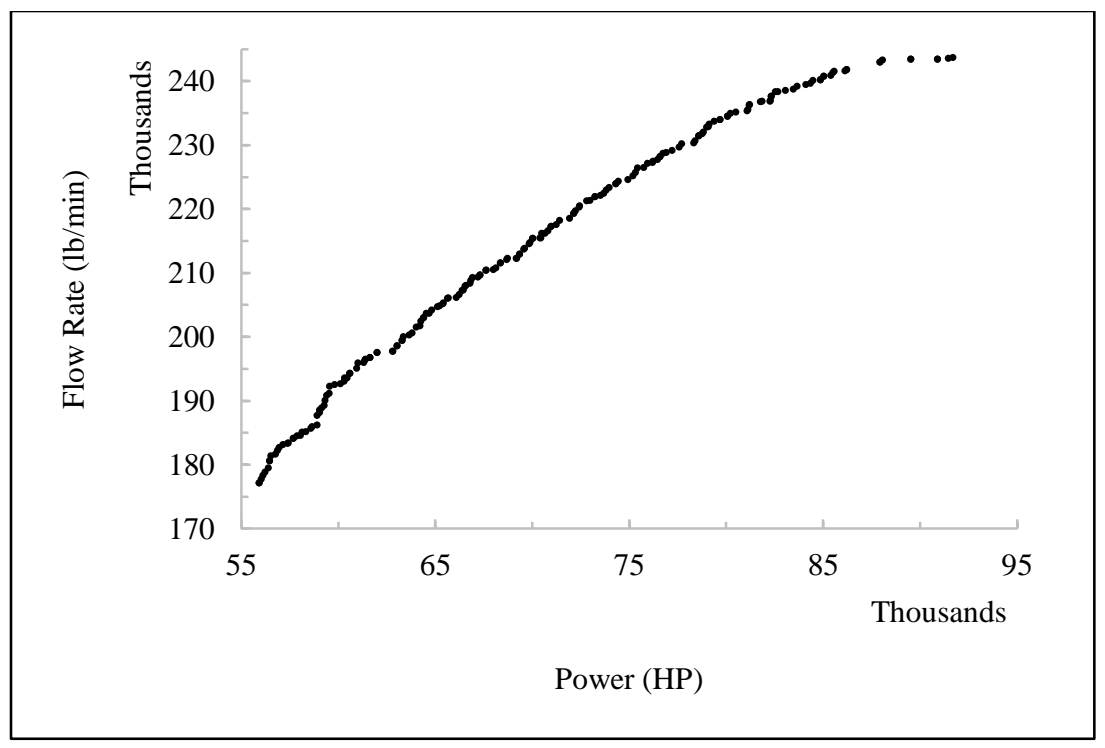

Figure 9. Optimum points for minimizing power consumption and maximizing gas delivery flow rate for branched topology

Case 3 which is a looped topology considers 10 decision variables and 30 constraints to optimize the objective functions. The optimization problem is solved and the Pareto optimal points for gas delivery flow rate maximization and power consumption minimization are presented in Figure 10. The CPU computation time for this case is averaged as $6,211 \mathrm{~ms}$. 


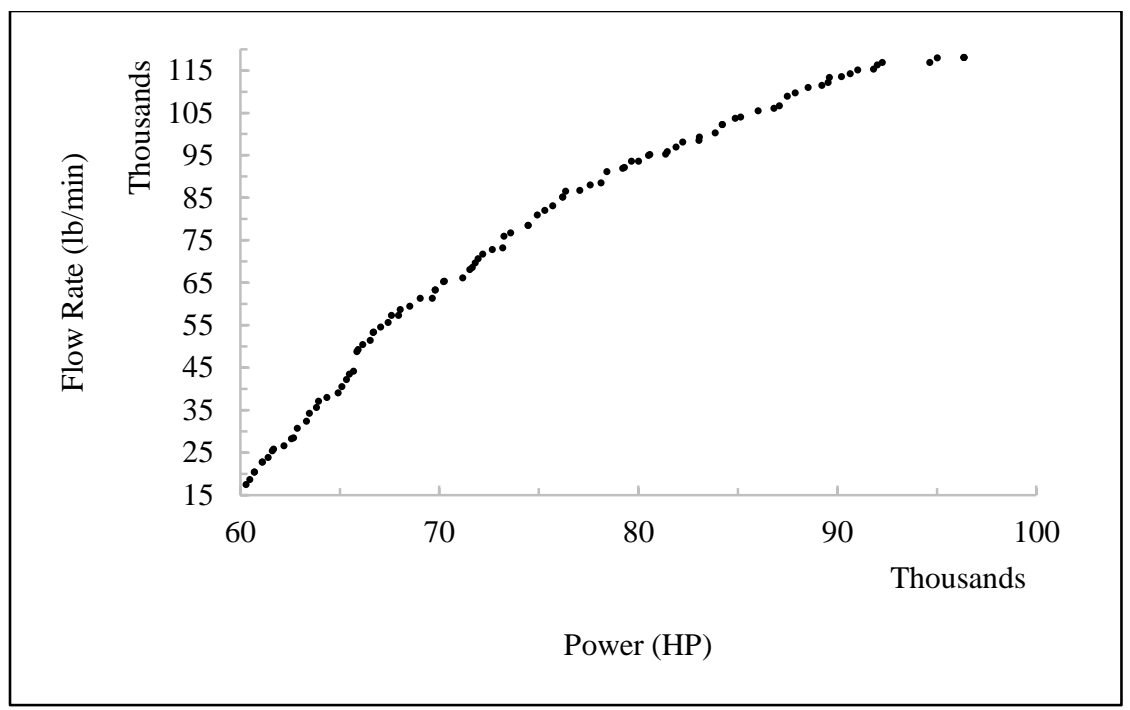

Figure 10. Optimum points for minimizing power consumption and maximizing gas delivery flow rate for looped topology

Generally, every Pareto optimal point is an acceptable solution. Table 2 shows selected Pareto optimal points for the linear topology and the variations of decision variables. Basically, a decision-maker has to choose a single solution from the optimal sets. In the natural gas industry, a decision maker is a person or group of persons who have better insight regarding the operation of the networks and the associated costs. During the selection of the best solution, the decision maker incorporates practical information and experience and identifies solutions which significantly improve the operation of the system. 
Table 2. Selected Pareto optimal points and variation of decision variables for Linear Topology

\begin{tabular}{|c|c|c|c|c|c|c|c|c|c|}
\hline \multicolumn{8}{|c|}{ Decision Variables } & \multicolumn{2}{|c|}{ Objective Functions } \\
\hline & \multicolumn{5}{|c|}{ Pressure(psia) } & \multicolumn{2}{|c|}{ Rotational Speed (rpm) } & \multirow{2}{*}{$\begin{array}{c}\text { Power (HP) } \\
\sum P\end{array}$} & \multirow{2}{*}{$\begin{array}{c}\text { Flow Rate } \\
\text { (lb/min) } \\
\sum b_{\text {out }}\end{array}$} \\
\hline $\mathrm{p}_{0}$ & $\mathrm{p}_{1}$ & $\mathrm{p}_{2}$ & $\mathrm{p}_{3}$ & $\mathrm{p}_{4}$ & $\mathrm{p}_{5}$ & $\omega_{1}$ & $\omega_{2}$ & & \\
\hline 648 & 621 & 683 & 662 & 752 & 738 & 7825 & 7200 & 53371 & 157720 \\
\hline 648 & 621 & 684 & 662 & 752 & 736 & 7639 & 7200 & 55477 & 164853 \\
\hline 655 & 624 & 687 & 663 & 747 & 729 & 8302 & 7807 & 56652 & 175118 \\
\hline 655 & 621 & 690 & 662 & 748 & 727 & 8297 & 7844 & 63090 & 185281 \\
\hline 664 & 628 & 694 & 663 & 752 & 729 & 9165 & 8652 & 66378 & 195472 \\
\hline 663 & 622 & 695 & 661 & 755 & 729 & 9389 & 9297 & 75239 & 205358 \\
\hline 666 & 621 & 684 & 643 & 755 & 726 & 9400 & 9399 & 84517 & 217030 \\
\hline 667 & 613 & 687 & 642 & 756 & 724 & 9399 & 9398 & 97888 & 227953 \\
\hline 660 & 601 & 691 & 642 & 760 & 726 & 9387 & 9400 & 116730 & 237767 \\
\hline
\end{tabular}

\subsection{Sensitivity Analysis and Discussion}

Sensitivity analysis is conducted to determine the impact of the change of the parameters. Figure 11 shows the nonlinear relationship between the volumetric flow rate of the gas and the pressure drop in a pipeline. It can be noted that the flow rate increases as the pressure drop in the pipeline increases. Figure 12 demonstrates a linear relation between the angular speed of a compressor and the compressor flow rate. It signifies the fact that as the speed of the compressor increases, the flow rate increases. 


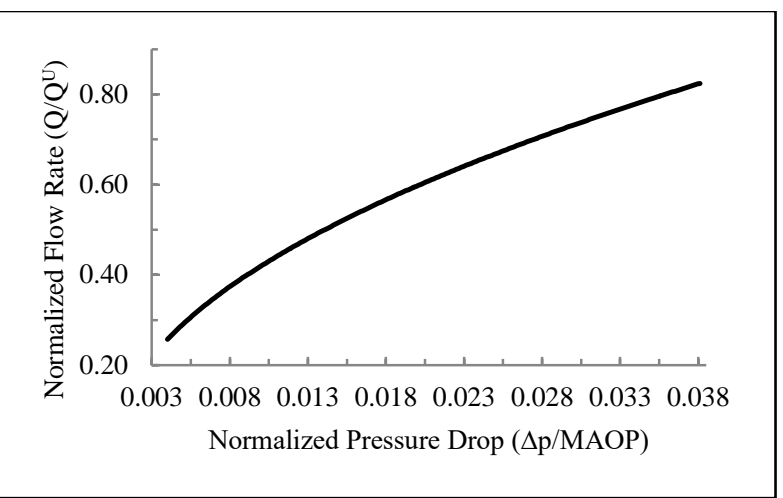

Figure 11. Sensitivity Analysis: Pressure drop vs. Volumetric Flow Rate

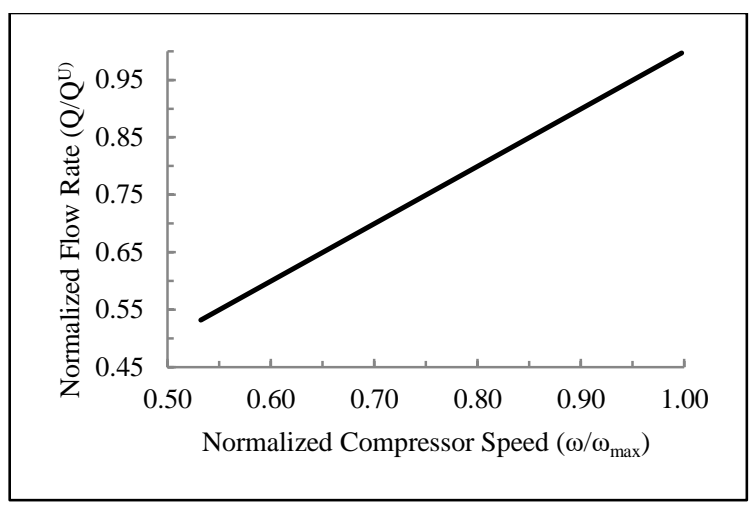

Figure 12.Sensitivity Analysis: Speed vs.

Figure 13 presents the relation between power consumed by the compressor station and the flow rate considering the effect of variations of the number of running compressors. It shows that higher flow rates require running more compressors. However, for the same amount of flow rate, running more compressors saves power.

By taking sample compression ratio points in the design domain of the compressors, the power consumed versus the flow rate of the compressor is plotted in Figure 14. It is observed that the higher the compression ratio, the higher the power demand of the compressor. Unfortunately, for pipeline system design engineer, lower compressor ratio means more compressor stations along the line. Practically, in operating pipeline, the compression ratio should be kept higher than the specified minimum value, otherwise, forward flow is impossible. 


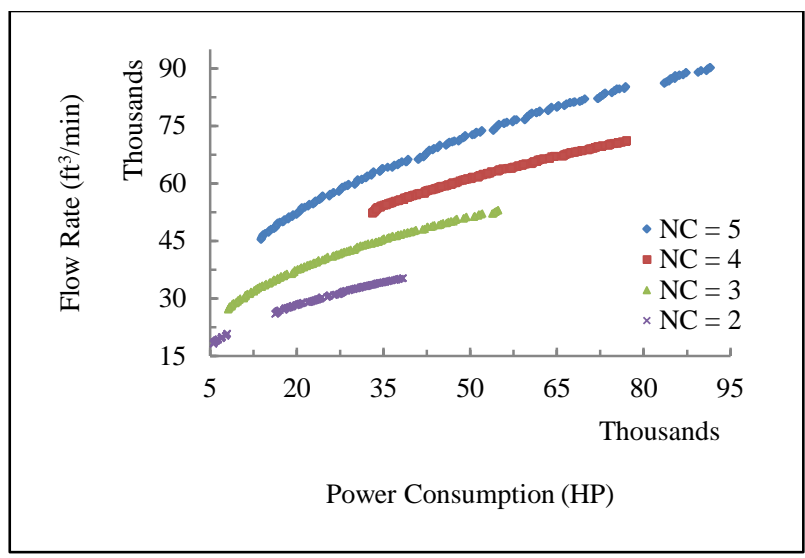

Figure 13. Sensitivity Analysis: Power / Flow Rate vs. Number of Compressors

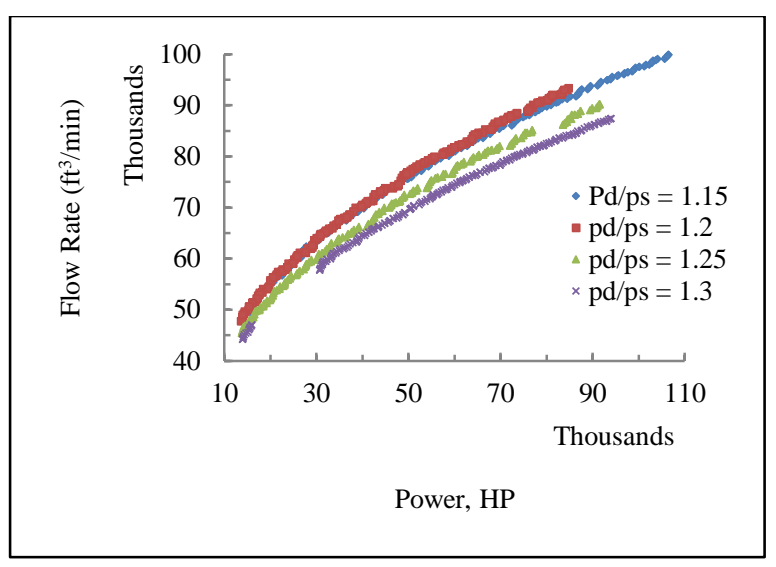

Figure 14. Sensitivity Analysis: Power /

Flow Rate vs. Compression Ratio

Figure 15 demonstrates relation between the power consumed and the flow rate of a pipeline for different suction pressures by considering sampling points between the minimum and maximum allowable pressure of the pipeline. It is observed that for the same flow rate, a slightly higher power is required when the suction pressure increases.

Finally, Figure 16 presents the relation between the power consumed and the flow rate of a pipeline for different rotating speeds of compressors. It can be noted that higher flow rates require more power at higher rotating speed of compressors. 


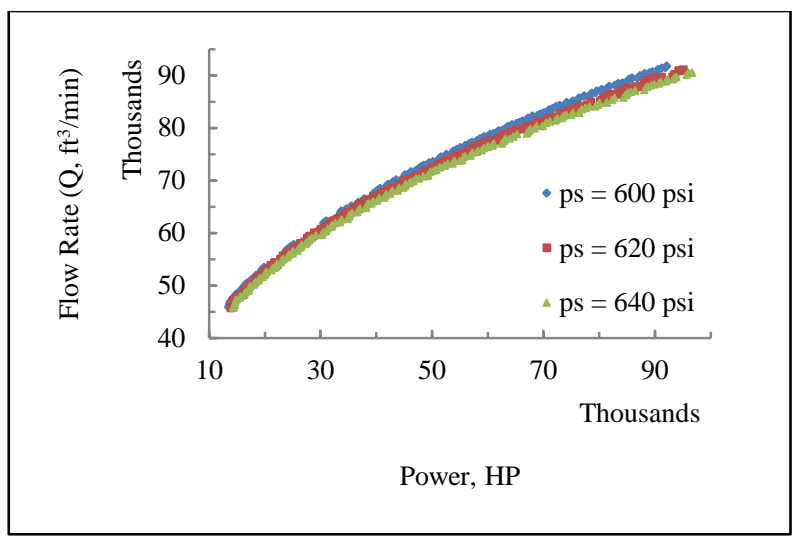

Figure 15. Power / Flow Rate vs. Suction

Pressure

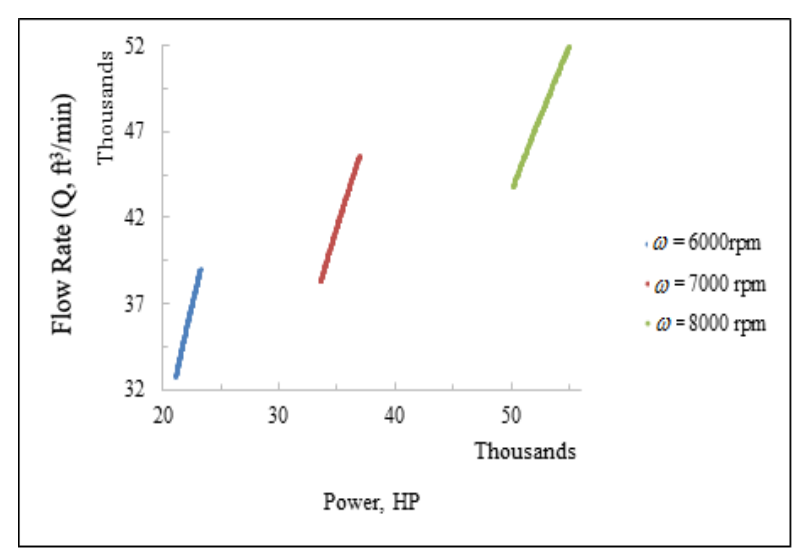

Figure 16. Sensitivity Analysis: Power / Flow

Rate vs. Speed Ratio

\section{Conclusion}

This paper established a multi-objective optimization model for natural gas transmission network. The developed model was applied to linear, branched and looped topologies. The goal was to find optimal operating condition for any network. The objectives considered are maximizing the gas delivery flow rate of the system and minimizing the power consumption of compressor stations subjected to pipeline and compressor constraints. Two types of continuous decision variables were considered, i.e. pressure at the nodes and rotational speed of compressors. The nature of the model is non-convex and non-linear. The case studies represent the most common topologies that exist in the real world system. They lay the foundation for investigating large and complex networks which are the combination of these three typical topologies. NSGA-II algorithm was used to solve the optimization problem. The model is solved using jMetal which is an objectoriented Java-based framework for solving multi-objective optimization problems. The solution of the multi-objective model is a set of non-dominated front solution points which is a trade-off curve between the two objectives. Generally, every non-dominated solution is an acceptable 
solution. However, a decision-maker has to choose a single solution from the optimal sets by incorporating practical information and experience which significantly improves the operation of

the system. Sensitivity analysis was performed to determine the impact of the change of parameters. As future work, research on large scale network optimization and additional objectives will be pursued.

\section{Acknowledgment}

This project is partially supported by National Science Foundation grants \#1140457, \#1457880 and \#1608886. Their support is greatly appreciated.

\section{References}

Alinia Kashani, Amir Hesam, and Reza Molaei. 2014. "Techno-Economical and Environmental Optimization of Natural Gas Network Operation." Chemical Engineering Research and Design 92 (11): 2106-22. doi:10.1016/j.cherd.2014.02.006.

Babonneau, Frédéric, Yurii Nesterov, and Jean-Philippe Vial. 2012. "Design and Operations of Gas Transmission Networks." Operations Research 60 (1): 34-47. doi:10.1287/opre.1110.1001.

Borraz-Sánchez, C., and R. Z. Ríos-Mercado. 2005. "A Hybrid Meta-Heuristic Approach for Natural Gas Pipeline Network Optimization." Lecture Notes in Computer Science 3636: 5465. http://www.scopus.com/inward/record.url?eid=2-s2.026944486202\&partnerID=tZOtx3y1.

Borraz-Sánchez, Conrado, and Dag Haugland. 2011. "Minimizing Fuel Cost in Gas Transmission Networks by Dynamic Programming and Adaptive Discretization." In Computers and Industrial Engineering, 61:364-72. doi:10.1016/j.cie.2010.07.012.

Borraz-Sanchez, Conrado, and Roger Z. Rios-Mercado. 2004. "A Non-Sequential Dynamic Programming Approach for Naturalgas Network Optimization." WSEAS Transactions on Systems 3 (4): 1384--1389.

Carter, R. G., J. M. Gablonsky, A. Patrick, C. T. Kelley, and O. J. Eslinger. 2000. “Algorithms for Noisy Problems in Gas Transmission Pipeline Optimization." Optimization and Engineering 2, no. 2: 139-57.

Chebouba, A., F. Yalaoui, A. Smati, L. Amodeo, K. Younsi, and A. Tairi. 2009. "Optimization of Natural Gas Pipeline Transportation Using Ant Colony Optimization.” Computers \& Operations Research 36 (6): 1916-23.

Christo, Angelo Tozi, Gilberto Mazoco Jubini, José Arica, and Marcia Miranda Azeredo. 2008. "An Approximation for Fuel Cost Minimization of the Steady State Gas Pipeline Network." 
In . XIV International Conference on Industrial Engineering and Operations Management.

Cobos-Zaleta, Diana, and Roger Z. Ríos-Mercado. 2002. "A MINLP Model for Minimizing Fuel Consumption on Natural Gas Pipeline Networks." In XI Latin-Ibero-American Conference on Operations Research, 27-31.

Deb, Kalyanmoy. 2001. "Multi-Objective Optimization Using Evolutionary Algorithms." John Wiley \& Sons, LTD. doi:10.1109/TEVC.2002.804322.

Demissie, A., and W. Zhu. 2015. "A Survey on Gas Pipelines Operation and Design Optimization Keywords :" In A Survey on Gas Pipelines Operation and Design Optimization, 734-43.

Durillo, Juan J., and Antonio J. Nebro. 2011. "JMetal: A Java Framework for Multi-Objective Optimization." Advances in Engineering Software 42 (10): 760-71. doi:10.1016/j.advengsoft.2011.05.014.

EIA. 2007. "Natural Gas Compressor Stations on the Interstate Pipeline Network." https://www.eia.gov/pub/oil_gas/natural_gas/analysis_publications/ngcompressor/ngcompre ssor.pdf.

Flores-Villarreal, Humberto J., and Roger Z. Ríos-Mercado. 2003. "Efficient Operation of Natural Gas Pipeline Networks: Computational Finding of High Quality Solutions.” In International Applied Business Research Conference, 1-7.

Hamedi, Maryam, Reza Zanjirani Farahani, and Gholamreza Esmaeilian. 2011. "19 Optimization in Natural Gas Network Planning." In Logistics Operations and Management, 393-420. doi:http://dx.doi.org/10.1016/B978-0-12-385202-1.00019-0.

Hernandez-Rodriguez, Guillermo. 2011. "Multiobjective Optimization of Natural Gas Transportation Networks." Institut National Polytechnique de Toulouse.

Kim, Seongbae, Roger Z. Ríos-Mercado, and E. Andrew Boyd. 2000. "Heuristics for Minimum Cost Steady-State Gas Transmission Networks." In Computing Tools for Modeling, Optimization and Simulation 12. Springer US: 203-13.

Liu, Enbin, Changjun Li, and Yi Yang. 2014. "Optimal Energy Consumption Analysis of Natural Gas Pipeline.” The Scientific World Journal 8.

Menon, Sashi E. 2005. Gas Pipeline Hydraulics. System. doi:10.1201/9781420038224.

Osiadacz, A. J., and M. Chaczykowski. 2001. "Comparison of Isothermal and Non-Isothermal Pipeline Gas Flow Models.” Chemical Engineering Journal 81 (1-3): 41-51. doi:10.1016/S1385-8947(00)00194-7.

Ríos-Mercado, Roger Z. 2002. "Natural Gas Pipeline Optimization.” Handbook of Applied Optimization, 3.

Ríos-Mercado, Roger Z., and Conrado Borraz-Sánchez. 2015. "Optimization Problems in Natural Gas Transportation Systems: A State-of-the-Art Review.” Applied Energy. doi:10.1016/j.apenergy.2015.03.017.

Sanaye, Sepehr, and Javad Mahmoudimehr. 2012. "Minimization of Fuel Consumption in Cyclic and Non-Cyclic Natural Gas Transmission Networks: Assessment of Genetic Algorithm Optimization Method as an Alternative to Non-Sequential Dynamic Programing." Journal of the Taiwan Institute of Chemical Engineers 43 (6): 904-17. doi:10.1016/j.jtice.2012.04.010.

Stoffregen, Jeff, Kamal K. Botros, Daniel J. Sennhauser, Krystyna Jungowski, and Hossein Golshan. 2005. "Pipeline Network Optimization-Application of Genetic Algorithm Methodologies." In PSIG Annual Meeting.

Tabkhi, F. 2007. "Optimization of Gas Transmission Networks." Institut National Polytechnique 
de Toulouse.

Tabkhi, Firooz, Luc Pibouleau, Guillermo Hernandez-Rodriguez, Catherine Azzaro-Pantel, and Serge Domenech. 2010. "Improving the Performance of Natural Gas Pipeline Networks Fuel Consumption Minimization Problems.” AIChE 56 (4): 946--964.

Villalobos-Morales, Yanet, Diana Cobos-Zaleta, and Conrado Borraz-Sánchez Roger RíosMercado Humberto J. Flores-Villarreal. 2003. “On NLP and MINLP Formulations and Preprocessing for Fuel Cost Minimization of Natural Gas Transmission Networks." In $S F$ Design, Service and Manufacturing Grantees and Research Conference.

Villalobos, Y., and R. Z. Ríos-Mercado. 2002. "Approximating the Fuel Consumption Function on Natural Gas Centrifugal Compressors." In NSF Design, Service, Manufacturing and Industrial Innovation Research Conference. San Juan, Puerto Rico.

Wolf, Daniel De, and Yves Smeers. 2000. "The Gas Transmission Problem Solved by an Extension of the Simplex Algorithm.” Management Science 46 (October 2014): 1454-65. doi:10.1287/mnsc.46.11.1454.12087.

Wu, Suming, R. Z. Ríos-Mercado, E. A. Boyd, and L. R. Scott. 2000. "Model Relaxations for the Fuel Cost Minimization of Steady-State Gas Pipeline Networks." Mathematical and Computer Modelling 31 (2-3): 197-220. doi:10.1016/S0895-7177(99)00232-0.

Wu, Xia, Changjun Li, Wenlong Jia, and Yufa He. 2014. "Optimal Operation of Trunk Natural Gas Pipelines via an Inertia-Adaptive Particle Swarm Optimization Algorithm." Journal of Natural Gas Science and Engineering 21: 10-18. doi:10.1016/j.jngse.2014.07.028.

Xu, Yuan, Wu Liu, Chunyuan Shi, and Wei Li. 2014. "Operation Optimization Method on Saving Compressor Energy of Natural Gas Pipeline.” In Bridges 10.

Zheng, Qipeng P, Steffen Rebennack, Niko A Iliadis, and Panos M Pardalos. 2010. "Optimization Models in The Natural Gas Industry." In Handbook of Power Systems I, 121-48. doi:10.1007/978-3-642-02493-1_6. 